\title{
Conductance of Rashba spin-split systems with ferromagnetic contacts
}

\author{
Morten Høgsbro Larsen, A. Mathias Lunde, and Karsten Flensberg \\ Ørsted Laboratory, Niels Bohr Institute fAPG, \\ Universitetsparken 5, 2100 Copenhagen, Denmark.
}

(Dated: November 1, 2018)

\begin{abstract}
We study theoretically the conductance of heterostructures with ferromagnetic conductors (F) and a two dimensional electron gas with Rashba spin-orbit interaction (R) using the Landauer-Büttiker formalism. Assuming a one-dimensional model, we first find the $S$-matrix for the FR interface. This result is then applied to different devices such as a FRF structure, first suggested by Datta and Das[Appl. Phys. Lett. 56, 665 (1990)]. We find analytic results for the conductance for the case of collinear magnetization.
\end{abstract}

\section{INTRODUCTION}

In 1990 Datta and Das showed that a spin-valve effect arises when a conductor with spin-orbit coupling of the Rashba type is connected to two ferromagnetic contacts. If furthermore the spin-orbit interaction can be tuned by an external electric field this effect can maybe be used as a spin transistor device. 1 The recent years advances in technology of spin injection into semiconductors has renewed the interest in this type of device. The Rashba spin-orbit coupling has been measured in a num ber of materials, e.g. in heterostructures based on InAs 2 or HgTe. 3 Also reports on electric field control of the Rashba interaction has been reported, 1 but there is stil some discussion about the interpretation of the results 5

In this paper we study the conductance of an electron gas with Rashba spin-orbit interaction ( $\mathrm{R}$ ) sandwiched between ferromagnetic $(\mathrm{F})$ or non-magnetic materials $(\mathrm{N})$ using a convenient $S$-matrix formalism. Throughout the text we restrict ourselves to a one-dimensional model. This could be realized by making a point contact structure defined by a set of split gates on top of the Rashba spin-split 2 dimensional electron gas (2DEG). In higher dimensions the interference effect due to the Rashba spinorbit coupling becomes weaker because the phase shifts depend on the length and the angle of the path between the two contacts.

\section{THE MODEL SYSTEM}

In this section we specify the Hamiltonian and the eigenstates for the F, R and $\mathrm{N}$ segments. The geometry is as shown in Fig. 1. The 2DEG has Rashba spinorbit scattering corresponding to an electric field in the $z$-direction. The Hamiltonians for the disorder free regions $\mathrm{F}, \mathrm{R}$, and $\mathrm{N}$ are, respectively,

$$
\begin{aligned}
\widehat{H}_{\mathrm{N}} & =\frac{\hat{p}^{2}}{2 m_{\mathrm{N}}}+E_{0}^{N}, \\
\widehat{H}_{\mathrm{F}} & =\frac{\hat{p}^{2}}{2 m_{\mathrm{F}}}+\Delta \frac{\mathbf{M}}{M} \cdot \widehat{\boldsymbol{\sigma}}+E_{0}^{F}, \\
\widehat{H}_{\mathrm{R}} & =\frac{\hat{p}^{2}}{2 m_{\mathrm{R}}}+\frac{\alpha}{\hbar}(\widehat{\mathbf{p}} \times \mathbf{E}) \cdot \widehat{\boldsymbol{\sigma}}+E_{0}^{R} .
\end{aligned}
$$

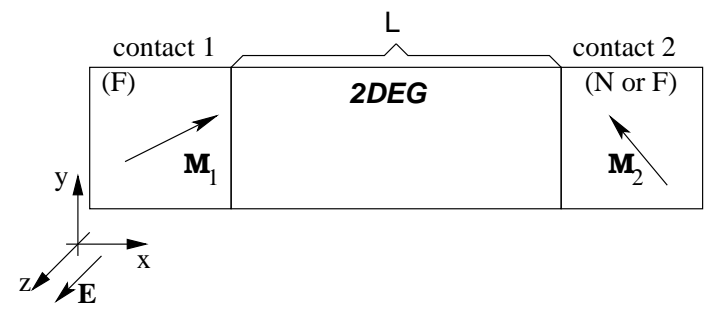

FIG. 1: Geometry of the structure studied in this paper.

Here we have defined the spin splitting energy in the ferromagnet $\Delta$ and the magnetization as $\mathbf{M}=$ $M(\sin \theta \cos \phi, \sin \theta \sin \phi, \cos \theta)$, with $\theta$ and $\phi$ being the usual spherical angles. The magnetizations in two contacts, $M_{1}$ and $M_{2}$, are in general different. Furthermore, $E_{0}^{(F, R, N)}$ is the band off-sets and we have used the parabolic band approximation and defined the effective masses by $m_{\mathrm{F}}, m_{\mathrm{R}}$ and $m_{\mathrm{N}}$. The last term is the Rashba spin orbit interaction where $\alpha$ is the Rashba interaction parameter, and $\mathbf{E}$ is the field that induces the spin-orbit coupling. The parameter $\alpha|E|$, which gives the strengths of the coupling, has been argued to be of order $\alpha|E| \sim 10^{-11} \mathrm{eVm}$ 2, 4

In order to solve the scattering problem we need to find the velocity operators. We obtain for the geometry as in Fig. 1

$$
\widehat{v}_{\mathrm{R}}=\frac{\widehat{p}}{m_{\mathrm{R}}}-\frac{\alpha E \widehat{\sigma}_{y}}{\hbar}, \quad \widehat{v}_{\mathrm{F}}=\frac{\widehat{p}}{m_{\mathrm{F}}}, \quad \widehat{v}_{\mathrm{N}}=\frac{\widehat{p}}{m_{\mathrm{N}}} .
$$

In $\mathrm{R}$ the velocity is modified due to the presence of the spin-orbit term. The velocity operator is derived for example by noting that $\widehat{v}=i[H, x] / \hbar$ or by using the Hamilton equation $\widehat{v}=\partial_{\widehat{p}} \widehat{H}$.

Next we find the eigenstates of the Hamiltonians (1)(3) with energy $\varepsilon$

$$
\begin{array}{ll}
\psi_{\mathrm{F} \sigma}^{ \pm}(x)=\phi_{\mathrm{F} \sigma}^{ \pm}(x)|\sigma\rangle, & \phi_{\mathrm{F} \sigma}^{ \pm}(x)=\sqrt{\frac{m_{\mathrm{F}}}{\hbar k_{\sigma}}} e^{ \pm i k_{\sigma} x} \\
\psi_{\mathrm{R} s}^{ \pm}(x)=\phi_{\mathrm{R} s}^{ \pm}(x)|s\rangle, & \phi_{\mathrm{R} s}^{ \pm}(x)=\frac{1}{\sqrt{v_{\mathrm{R}}}} e^{ \pm i k_{s} x} \\
\psi_{\mathrm{N} \mu}^{ \pm}(x)=\phi_{\mathrm{N} \mu}^{ \pm}(x)|\mu\rangle, & \phi_{\mathrm{N} \mu}^{ \pm}(x)=\sqrt{\frac{m}{\hbar k}} e^{ \pm i k x}
\end{array}
$$


where the spinor eigenstates are given by

$$
\begin{aligned}
\{|\sigma\rangle,|\bar{\sigma}\rangle\} & =\frac{1}{\sqrt{2(1 \pm \cos \theta)}}\left(\begin{array}{c}
\cos \theta \pm 1 \\
\sin \theta e^{i \phi}
\end{array}\right), \\
\{|s\rangle,|\bar{s}\rangle\} & =\frac{1}{\sqrt{2}}\left(\begin{array}{c} 
\pm i \\
1
\end{array}\right), \\
\{|\mu\rangle,|\bar{\mu}\rangle\} & =\left\{\left(\begin{array}{l}
1 \\
0
\end{array}\right),\left(\begin{array}{l}
0 \\
1
\end{array}\right)\right\},
\end{aligned}
$$

and the relations between the wavenumbers and the eigenenergy $\varepsilon$ are

$$
\begin{aligned}
\varepsilon & =\frac{\hbar^{2} k_{\sigma}^{2}}{2 m_{\mathrm{F}}}+\sigma \Delta+E_{0}^{F} \\
\varepsilon & =\frac{\hbar^{2}}{2 m_{\mathrm{R}}}\left[k_{s}\left(k_{s}+2 s k_{\mathrm{R}}\right)\right]+E_{0}^{R}, \\
\varepsilon & =\frac{\hbar^{2} k^{2}}{2 m_{\mathrm{N}}}+E_{0}^{N}
\end{aligned}
$$

Here $s$ and $\sigma$ is \pm 1 and we have defined

$$
k_{\mathrm{R}}=\frac{\alpha E m_{\mathrm{R}}}{\hbar^{2}} .
$$

The velocity in the Rashba material $v_{\mathrm{R}}$ is

$$
v_{\mathrm{R}}=\frac{\hbar}{m_{\mathrm{R}}}\left(k_{s}+s k_{R}\right)=\frac{\hbar}{m_{\mathrm{R}}}\left[\frac{2 m_{\mathrm{R}}\left(\varepsilon-E_{0}^{R}\right)}{\hbar^{2}}+k_{\mathrm{R}}^{2}\right]^{1 / 2},
$$

where the last equality follows from Eq. (12). Note that the velocity is independent of the Rashba spin direction.

\section{SETTING UP THE $S$-MATRIX FOR INTERFACE BETWEEN F AND $R$}

Now we consider the scattering problem related to an interface between an $\mathrm{F}$ and an $\mathrm{R}$ region. We start by considering a scattering state for an interface without disorder and with an electron with spin $\sigma$ incoming from the ferromagnetic side. This we use to prove that the scattering problem becomes diagonal in the spin degree of freedom. The scattering state is defined by

$$
\psi_{R F, \sigma}(x)=\left\{\begin{array}{cl}
\phi_{F \sigma}^{+}(x)|\sigma\rangle+r_{\sigma \sigma} \phi_{F \sigma}^{-}(x)|\sigma\rangle, & x \in F \\
\sum_{s} t_{\sigma \sigma} \alpha_{s \sigma} \phi_{R s}^{+}(x)|s\rangle, & x \in R
\end{array}\right.
$$

where we have $\mathrm{F}$ in $x<0$ and $\mathrm{R}$ in $x>0$.

We solve for the scattering state without including the possibility of being reflected or transmitted in an $|\bar{\sigma}\rangle$. This is possible because the spin states in $\mathrm{R}$ carry the same group velocity and any linear combination of $|s\rangle$ and $|\bar{s}\rangle$ is thus an eigenstate to the velocity operator. Therefore we can choose the coefficients $\alpha_{s \sigma}$ such that the transmitted state at the interface, $x=0$, is equal to the state $|\sigma\rangle$

$$
\sum \alpha_{s \sigma}|s\rangle=|\sigma\rangle \Rightarrow \alpha_{s \sigma}=\langle s \mid \sigma\rangle \equiv U_{s \sigma}
$$

The continuity conditions at the interface are that the wavefunction and the probability currents must be continuous

$$
\psi_{R F}\left(0^{-}\right)=\psi_{R F}\left(0^{+}\right), \quad \widehat{v}_{\mathrm{F}} \psi_{R F}\left(0^{-}\right)=\widehat{v}_{\mathrm{R}} \psi_{R F}\left(0^{+}\right) .
$$

These 4 equations reduce to only 2 linearly independent equations because, as mentioned above, the $\psi_{R F}$ is eigenstate of $\widehat{v}_{R}$, and we obtain

$$
\begin{aligned}
\frac{1}{\sqrt{v_{\sigma}}}\left(1+r_{\sigma \sigma}\right) & =\frac{1}{\sqrt{v_{\mathrm{R}}}} t_{\sigma \sigma} \\
\sqrt{v_{\sigma}}\left(1-r_{\sigma \sigma}\right) & =\sqrt{v_{\mathrm{R}}} t_{\sigma \sigma} .
\end{aligned}
$$

This set of equations corresponds to the scattering between two metals with Fermi velocities given by $v_{\sigma}$ and $v_{\mathrm{R}}$, respectively. The are readily solved

$$
t_{\sigma \sigma}=\frac{2}{\sqrt{\frac{v_{\sigma}}{v_{\mathrm{R}}}}+\sqrt{\frac{v_{\mathrm{R}}}{v_{\sigma}}}}, \quad r_{\sigma \sigma}=\frac{\sqrt{\frac{v_{\sigma}}{v_{\mathrm{R}}}}-\sqrt{\frac{v_{\mathrm{R}}}{v_{\sigma}}}}{\sqrt{\frac{v_{\sigma}}{v_{\mathrm{R}}}}+\sqrt{\frac{v_{\mathrm{R}}}{v_{\sigma}}}} .
$$

Here $t_{\sigma \sigma}$ and $r_{\sigma \sigma}$ are the transmission and reflection amplitudes written in the basis defined by the spin eigenstates in $\mathrm{F}$. The transmission and reflection matrices is thus diagonal in this basis

$$
t_{\sigma \sigma^{\prime}}=t_{\sigma \sigma} \delta_{\sigma \sigma^{\prime}}, \quad r_{\sigma \sigma^{\prime}}=r_{\sigma \sigma} \delta_{\sigma \sigma^{\prime}}
$$

When we want to combine the transmission through the $\mathrm{FR}$ interface with propagation in $\mathrm{R}$, it is more convenient to write the transmission and reflection matrices in the basis set defined by spin eigenstates in $R$. These we denote by $\mathbf{t}^{R}$ and $\mathbf{r}^{R}$ and they are

$$
\begin{aligned}
& t_{s^{\prime} s}^{R}=\sum_{\sigma}\left\langle s^{\prime} \mid \sigma\right\rangle t_{\sigma \sigma}\langle\sigma \mid s\rangle \quad \text { or } \quad \mathbf{t}^{R}=\mathbf{U} \mathbf{t} \mathbf{U}^{\dagger}, \\
& r_{s^{\prime} s}^{R}=\sum_{\sigma}\left\langle s^{\prime} \mid \sigma\right\rangle r_{\sigma \sigma}\langle\sigma \mid s\rangle \quad \text { or } \quad \mathbf{r}^{R}=\mathbf{U r} \mathbf{U}^{\dagger} .
\end{aligned}
$$

Next we consider transmission from $\mathrm{R}$ to $\mathrm{F}$. For this we set up a scattering state where the incoming electron from the $\mathrm{R}$ side at $x=0$ has a definite spin with respect to the $\mathrm{F}$ side. For the same reasons as above we need only consider one $\mathrm{F}$ spin channel at a time. We thus write

$\psi_{F R, \sigma}(x)=\left\{\begin{array}{cl}\sum_{s}\left[\phi_{R s}^{-}(x)+r_{\sigma \sigma}^{\prime} \phi_{R s}^{+}(x)\right] \alpha_{s \sigma}|s\rangle, & x \in R \\ t_{\sigma \sigma}^{\prime} \phi_{F \sigma}^{-}(x)|\sigma\rangle, & x \in F\end{array}\right.$

Solving for $t_{\sigma \sigma}^{\prime} r_{\sigma \sigma}^{\prime}$ in the same way as above, we obtain the reverse transmission and reflection matrices written in the $\mathrm{F}$ spin basis as

$$
\mathbf{t}^{\prime}=\mathbf{t}, \quad \mathbf{r}^{\prime}=-\mathbf{r} .
$$

When converted into the basis corresponding to $\mathrm{R}$ eigenstates, this becomes

$$
\mathbf{t}^{\prime R}=\mathbf{U} \mathbf{t} \mathbf{U}^{\dagger}, \quad \mathbf{r}^{\prime R}=-\mathbf{U} \mathbf{r} \mathbf{U}^{\dagger} .
$$


As is evident from Eqs. (26) and the fact that $\mathbf{t r}=\mathbf{r t}$ the $S$-matrix for the FR interface

$$
\mathbf{S}=\left(\begin{array}{ll}
\mathbf{r} & \mathbf{t}^{\prime} \\
\mathbf{t} & \mathbf{r}^{\prime}
\end{array}\right)
$$

is indeed unitary.

The one-dimensional scattering problem is easily generalized to include disorder or interface scattering if the scattering conserves spin, because the scattering problem would still separate in two parts, one for each spin direction in the ferromagnet. The total $S$-matrix is then given by the corresponding problem of scattering between two disordered conductors with different Fermi velocities. In the following we do not specify the the transmission coefficient between $\mathrm{F}$ and $\mathrm{R}$, and the results are therefore general for any type of interface, as long as spin flip scattering is not present.

\section{LANDAUER-BÜTTIKER FORMULA}

If the contacts are made of ferromagnetic conductors with a partial polarization then the conductance is according to the Landuer-Büttiker formula given by

$$
G=\frac{e^{2}}{h} \sum_{\sigma_{L} \sigma_{R}}\left|t_{\sigma_{L} \sigma_{R}}\right|^{2}=\frac{e^{2}}{h} \operatorname{Tr}\left[\mathbf{t}^{\dagger} \mathbf{t}\right]
$$

where the $t_{\sigma_{L} \sigma_{R}}$ is the transmission amplitude through the entire structure written in a basis where the left (rigth) spin state is labeled by $\sigma_{L(R)}$. This result is of course independent of spin basis set. If, however, we consider a situation where one or both of the contacts are half metallic, i.e. the polarization is $100 \%$, then summation over final and initial states of the transmission matrix $t_{\sigma_{L} \sigma_{R}}$ must be restricted. This means that the sum over $\sigma_{L} \sigma_{R}$ in Eq. (29), must include only populated spin states.

Since we are mainly interested in the the spin dependent transmission we consider the situation where we can neglect multiple reflections at the interfaces and thus only calculate the conductance to lowest order in the transmission, i.e.,

$$
\mathbf{t}^{(1)}=\mathbf{t}_{2}^{\prime} \mathbf{L} \mathbf{t}_{1}
$$

where $\mathbf{t}_{1}$ and $\mathbf{t}_{2}$ are the transmission amplitudes for the two interfaces and $\mathbf{L}$ describes the transmission through the middle region. We take the middle region to be ballistic but with a Rashba spin orbit coupling and hence

$$
\mathbf{L}^{R}=\left(\begin{array}{cc}
e^{i k_{+} L} & 0 \\
0 & e^{i k_{-} L}
\end{array}\right)
$$

If the coherence is longer than $L$ and if the resonant nature of the transmission is important one should instead use the formula for $\mathbf{t}$ to all orders in $\mathbf{L}$

$$
\mathbf{t}=\mathbf{t}_{2}^{\prime} \sum_{n=0}^{\infty}\left(\mathbf{L} \mathbf{r}_{1}^{\prime} \mathbf{L}^{\prime} \mathbf{r}_{2}^{\prime}\right)^{n} \mathbf{L} \mathbf{t}_{1}=\mathbf{t}_{2}^{\prime}\left(1-\mathbf{L} \mathbf{r}_{1}^{\prime} \mathbf{L}^{\prime} \mathbf{r}_{2}^{\prime}\right)^{-1} \mathbf{L} \mathbf{t}_{1}
$$

\section{A. Conductance of a FRN structure}

As a first application of our formalism we will calculate the transmission properties and conductance for a FRN structure. The first-order expression for the transmission matrix is in the Rashba representation given by

$$
\mathbf{t}_{\mathrm{FRN}}^{(1) R}=\mathbf{t}_{2}^{\prime} \mathbf{L}^{R} \mathbf{U}_{1} \mathbf{t}_{1} \mathbf{U}_{1}^{\dagger}
$$

where $\left(\mathbf{t}_{2}^{\prime}\right)_{\sigma \sigma^{\prime}}=\delta_{\sigma \sigma^{\prime}} t_{2}$ and $\left(\mathbf{t}_{1}\right)_{\sigma \sigma^{\prime}}=\delta_{\sigma \sigma^{\prime}} t_{\sigma \sigma}$. Inserting this into the conductance formulae for the case of a partially polarized ferromagnetic contact

$$
G_{\mathrm{FRN}}^{(1)}=\frac{e^{2}}{h} \operatorname{Tr}\left[\left(\mathbf{t}_{\mathrm{FRN}}^{(1) R}\right)^{\dagger}\left(\mathbf{t}_{\mathrm{FRN}}^{(1) R}\right)\right]=\frac{e^{2}}{h}\left|t_{2}\right|^{2} \sum_{\sigma}\left|t_{\sigma \sigma}\right|^{2},
$$

which is independent of all angles. This is also the case for a fully polarized ferromagnetic contact where the conductance becomes

$$
G_{\mathrm{FRN}}^{100 \%}=\frac{e^{2}}{h} \sum_{s}\left|t_{\sigma s}\right|^{2}=\frac{e^{2}}{h}\left|t_{2}\right|^{2}\left|t_{\sigma \sigma}\right|^{2} .
$$

which is of course what we expected because the transmission from $\mathrm{F}$ to $\mathrm{R}$ was diagonal in $\mathrm{F}$ spin representation and the transmission between $\mathrm{R}$ and $\mathrm{N}$ is independent of spin direction. Here we have simply reproduced the result obtained by Ref. 6 .

The FRN is thus not sensitive to the phase shift induced by the Rashba interaction. The phase shift acts as a spin polarizer which depends on the length $L$. The way to detect the polarization is of course to replace the normal metal contact by a ferromagnetic confact, which was the original suggestion by Datta and Das 1 which we study in the following section.

\section{B. Conductance of a $\mathrm{FRF}^{\prime}$ structure}

As a second application we consider the transmission matrix for the Datta and Das structure, namely a FRF' device. The transmission to first order in the transmissions is $\mathbf{t}_{\mathrm{FRF}^{\prime}}^{R(1)}=\mathbf{t}_{2}^{\prime R} \mathbf{L}^{R} \mathbf{t}_{1}^{R}$, where $\mathbf{t}_{1}$ and $\mathbf{t}_{2}$ denote the transmission for the two interfaces and $\mathbf{L}^{\mathrm{R}}$ is the transmission matrix for the $\mathrm{R}$ system defined in Eq. (31). Inserting the results from the previous sections, one obtains

$$
\mathbf{t}_{\mathrm{FRF}^{\prime}}^{R(1)}=\mathbf{U}_{2} \mathbf{t}_{2}^{\prime} \mathbf{U}_{2}^{\dagger} \mathbf{L}^{R} \mathbf{U}_{1} \mathbf{t}_{1} \mathbf{U}_{1}^{\dagger} .
$$

If the ferromagnetic contacts are not $100 \%$ spin polarized the conductance is found by inserting this into Eq. (29). For the case where the two magnetizations are parallel, this is explicitly found to be

$$
\begin{array}{r}
G_{\mathrm{FRF}^{\prime}}^{(1)}(\|)=\frac{e^{2}}{h}[ \\
\frac{1}{2} T_{1} T_{2}+\frac{1}{2} S_{1} S_{2}\left(1-2 \sin ^{2} \delta\right. \\
\left.\left.+2 \sin ^{2}(\delta) \sin ^{2}(\phi) \sin ^{2}(\theta)\right)\right]
\end{array}
$$


where

$$
\begin{aligned}
& \delta=\frac{L\left(k_{\bar{s}}-k_{s}\right)}{2}, \\
& T_{1}=\left|t_{1, \sigma \sigma}\right|^{2}+\left|t_{1, \bar{\sigma} \bar{\sigma}}\right|^{2}, \quad S_{1}=\left|t_{1, \sigma \sigma}\right|^{2}-\left|t_{1, \bar{\sigma} \bar{\sigma}}\right|^{2},
\end{aligned}
$$

and similarly for $T_{2}$ and $S_{2}$.

We can now use the result in Eq. (36) to study some special cases. Suppose the two interfaces are equal and the magnetization of the two magnets are either pointing in the same direction $(\leftleftarrows)$ i.e. $T_{1}=T_{2}$ and $S_{1}=S_{2}$, or in opposite directions $(\rightleftarrows)$ which is equivalent to setting $\Delta_{1}=-\Delta_{2}$ in Eq. (11), i.e. $T_{1}=T_{2}$ and $S_{1}=-S_{2}$, then we get

$$
\begin{aligned}
G_{\mathrm{FRF}}^{(1)}(\leftleftarrows)=\frac{e^{2}}{h}[ & \frac{1}{2} T^{2}+\frac{1}{2} S^{2}\left(1-2 \sin ^{2} \delta\right. \\
& \left.\left.+2 \sin ^{2}(\delta) \sin ^{2}(\phi) \sin ^{2}(\theta)\right)\right],
\end{aligned}
$$

and $G_{\mathrm{FBF}}^{(1)}(\rightleftarrows)$ follows by the replacement $S^{2} \rightarrow-S^{2}$ in Eq. (38). The minimum conductance that one can achieve by tuning the Rashba coupling (through $\delta$ ) and/or the magnetization direction, is

$$
\left.G_{\mathrm{FRF}}^{(1)}\right|_{\min }=\frac{e^{2}}{h} \frac{1}{2}\left(T^{2}-|S|^{2}\right)=\frac{2 e^{2}}{h}\left|t_{\sigma \sigma}\right|^{2}\left|t_{\bar{\sigma} \bar{\sigma}}\right|^{2} .
$$

The maximum modulation we can have by changing $\delta$ or the angles of the magnitization is

$$
\left.\Delta G_{\mathrm{FRF}}^{(1)}\right|_{\max }=\frac{e^{2}}{h} S^{2}=\frac{e^{2}}{h}\left(T_{\sigma \sigma}-T_{\bar{\sigma} \bar{\sigma}}\right)^{2},
$$

which is proportional to the difference between the velocities in the ferromagnet.

The magnetoresponse is given by

$$
\begin{aligned}
\Delta r & \equiv \frac{G_{\mathrm{FRF}}^{(1)}(\leftleftarrows)-G_{\mathrm{FRF}}^{(1)}(\rightleftarrows)}{G_{\mathrm{FRF}}^{(1)}(\leftleftarrows)+G_{\mathrm{FRF}}^{(1)}(\rightleftarrows)} \\
& =\frac{S^{2}}{T^{2}}\left(\cos 2 \delta+2 \sin ^{2}(\delta) \sin ^{2}(\phi) \sin ^{2}(\theta)\right)
\end{aligned}
$$

For the case of two similar half-metallic contacts, i.e. $100 \%$ polarization, the conductance for parallel case is given by setting $T_{1}=T_{2}=S_{1}=S_{2}=t_{\sigma \sigma}^{2}$,

$$
\begin{aligned}
& G_{\mathrm{FRF}}^{(1)}(\leftleftarrows, 100 \%)=\frac{e^{2}}{h}\left|t_{\sigma \sigma}\right|^{2} \\
& \times\left(1-\sin ^{2} \delta+\sin ^{2}(\delta) \sin ^{2}(\phi) \sin ^{2}(\theta)\right),
\end{aligned}
$$

and the anti-parallel is given by setting $T_{1}=T_{2}=S_{1}=$ $-S_{2}=\left|t_{\sigma \sigma}\right|^{2}$,

$$
G_{\mathrm{FRF}}^{(1)}(\rightleftarrows, 100 \%)=\frac{e^{2}}{h}\left|t_{\sigma \sigma}\right|^{2} \sin ^{2} \delta\left(1-\sin ^{2}(\phi) \sin ^{2}(\theta)\right) .
$$

For the half-metallic case the maximan modulation is $t_{\sigma \sigma}^{2}$, and the magnetoresponse as defined in Eq. (41) becomes

$$
\Delta r=1-2 \sin ^{2} \delta+2 \sin ^{2}(\delta) \sin ^{2}(\phi) \sin ^{2}(\theta) .
$$

This expression shows that the magnetoresponse can be tuned between -1 and 1 depending on the Rashba coupling and the direction of the magnetization through $\phi$ and $\theta$.

\section{SUMMARY}

We found analytic result for the conductance of an FRF structure with collinear magnetization, Eq. (36). This formula shows that the minimum conductance is given by $G_{\min }=\left(e^{2} / h\right)\left|t_{1 \sigma}\right|^{2}\left|t_{2 \bar{\sigma}}\right|^{2}+\left|t_{1 \bar{\sigma}}\right|^{2}\left|t_{2 \sigma}\right|^{2}$, where $\left|t_{(1,2) \sigma}\right|^{2}$ and $\left|t_{(1,2) \bar{\sigma}}\right|^{2}$ are the transmission probabilities through the interface $(1,2)$ for the spin direction $\sigma$ and $\bar{\sigma}$, respectively. In order to have a substantial valve effect the contacts should therefore by half-metallic or the transmission coefficients must be strongly spin dependent.
1 S. Datta and B. Das, Appl. Phys. Lett. 56, 665 (1990)

2 T. Matsuyama, R. Kürsten, C. Meinser, and U. Merkt, Phys. Rev. B 61, 15588 (2000); Y. Sato, T. Kita, S. Gozu, and S. Yamada, J. Appl. Phys. 89, 8017 (2001).

${ }^{3}$ X.C. Zhang, A. Pfeuffer-Jeschke, K. Ortner, V. Hock, H. Buhrmann, C.R. Becker, G. Landwehr, Phys. Rev. B 63, 245305 (2001).
4 J. Nitta, T. Akazaki, H. Takayanagi, and T. Enoki, Phys. Rev. Lett. 78, 1335 (1997).

5 A.C.H. Rowe, J. Nehls, R.A. Strading, and R.S. Ferguson, Phys. Rev. B 63, R201307 (2001).

6 L.W. Molenkamp, G. Schmidt, and G.E. Bauer, Phys. Rev. B 64, R121202 (2001). 